\title{
Correction to: Androgen receptor regulates expression of skeletal muscle-specific proteins and muscle cell types
}

\author{
Saleh Altuwaijri ${ }^{1} \cdot$ Dong Kun Lee ${ }^{1} \cdot$ Kuang-Hsiang Chuang $^{1} \cdot$ Huei-Ju Ting ${ }^{1} \cdot$ Zhiming Yang $^{1} \cdot$ Qingquan Xu $^{1}$. \\ Meng-Yin Tsai ${ }^{2} \cdot$ Shuyuan Yeh ${ }^{1} \cdot$ LeRoy A. Hanchett ${ }^{1} \cdot$ Hong-Chiang Chang $^{3} \cdot$ Chawnshang Chang ${ }^{1}$
}

Published online: 2 July 2020

(c) Springer Science+Business Media, LLC, part of Springer Nature 2020

\section{Correction to: Endocrine}

https://doi.org/10.1385/ENDO:25:1:49

The original version of this article unfortunately contained an error in Fig. 2. The authors apologize for the error and assure that the correction of this image will not alter the conclusion of the results. The corrected figure is given below:
We apologize for the mistake during the figure preparation. We inadvertently used the same data for figure assembly. The corrected figure is provided in the bottom. We assure this correction does not change the original result and conclusion.

Chawnshang Chang

chang@urmc.rochester.edu

1 George Whipple Lab for Cancer Research, Departments of Pathology, Urology, and The Cancer Center, University of Rochester Medical Center, Rochester, New York 14642, USA

2 Department of OB/GYN, Chang Gang University, Kaohsiung 833, Taiwan

3 Department of Urology, National Taiwan University, Taipei 100, Taiwan 

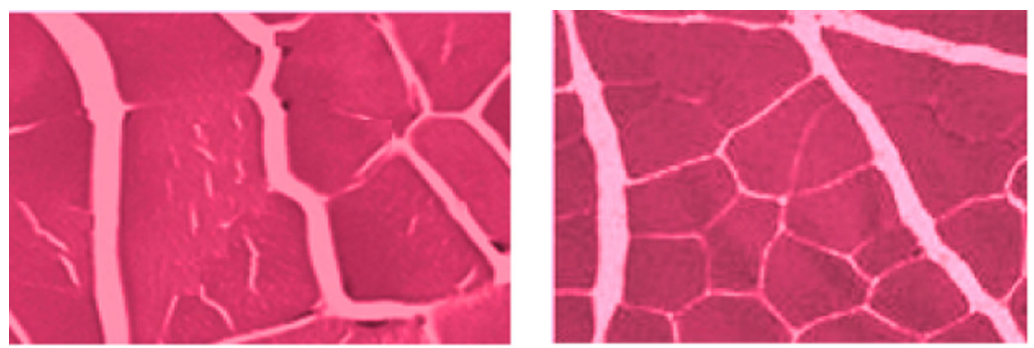

H/E staining
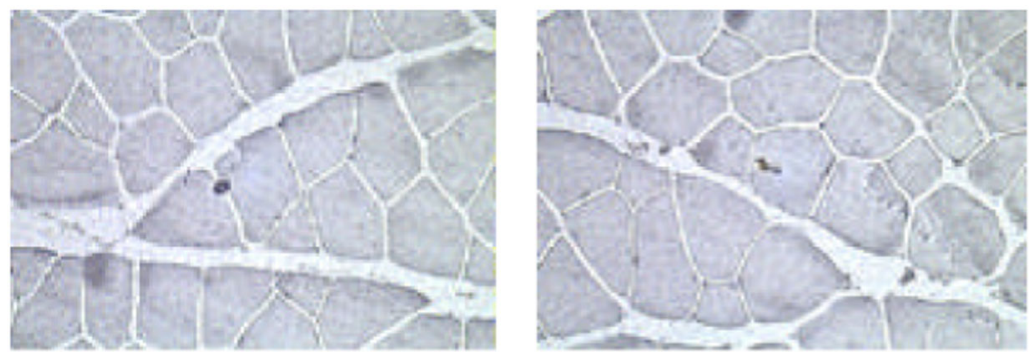

IHC (anti-desmin)

WT

ARKO

Fig. 2 